\title{
Pneumonia Identification Using Nursing Home Records
}

\author{
Sheryl Zimmerman, PhD; Lauren W. Cohen, MA; Kezia Scales, PhD; David Reed, PhD; \\ Christina Horsford, MSW, MPA; David J. Weber, MD, MPH; and Philip D. Sloane, MD, MPH
}

\begin{abstract}
Pneumonia is a leading cause of death among nursing home residents; consequently, prevention and treatment are important for quality improvement. To be pragmatic, quality improvement depends on sensitive case identification using nursing home records; however, no studies have examined the reliability of different methods of pneumonia case finding from records. The current authors compared three established strategies for defining pneumonia using records from 1,119 residents across 16 nursing homes: recorded diagnosis of pneumonia, modified McGeer criteria (chest x-ray infiltrate plus specified signs/symptoms), and antibiotic prescription plus pneumonia-specific signs. Chart diagnosis detected 107 cases, modified McGeer criteria detected 84 cases, and antibiotic prescription detected 47 cases. Diagnosis included all cases identified by the McGeer criteria and all but one case identified by antibiotic use. Based on findings, recorded diagnosis of pneumonia is a highly sensitive and pragmatic method to ascertain pneumonia in nursing homes, and is recommended for use in quality improvement and research.
\end{abstract}

[Res Gerontol Nurs. 2016; 9(3):109-114.]

Dr. Zimmerman is University Kenan Distinguished Professor, Schools of Social Work and Public Health, and Co-Director, Program on Aging, Disability, and Long-Term Care, Cecil G. Sheps Center for Health Services Research, Ms. Cohen is Research Associate, and Dr. Reed is Research Associate, Program on Aging, Disability, and Long-Term Care, Cecil G. Sheps Center for Health Services Research, Ms. Horsford is Doctoral Student, School of Social Work, Dr. Weber is Professor, Department of Medicine, School of Medicine, and Dr. Sloane is Elizabeth and Oscar Goodwin Distinguished Professor, Department of Family Medicine, School of Medicine, and Co-Director, Program on Aging, Disability, and Long-Term Care, Cecil G. Sheps Center for Health Services Research, University of North Carolina at Chapel Hill, Chapel Hill; and Dr. Scales is Post-Doctoral Fellow, School of Nursing, Duke University, Durham, North Carolina.

Dr. Weber has received honoraria and serves as a speaker and consultant on matters related to infections. The remaining authors have disclosed no potential conflicts of interest, financial or otherwise. This research was supported by grant R01HS022298 from the Agency for Healthcare Research and Quality. The sponsor provided support for the design and conduct of the study; collection, management, analysis, and interpretation of the data; and preparation of the manuscript; the sponsor was not involved in the decision to submit the paper for publication. The authors thank the staff and residents of the nursing homes who participated in this project and in the Collaborative Studies of Long-Term Care. It is because of their ongoing partnership that we can learn about the quality of care and quality of life in nursing homes and other residential settings and work together to improve both when indicated. The authors also thank Kimberly Ward, BA, for her expert data collection.

Address correspondence to Sheryl Zimmerman, PhD, University Kenan Distinguished Professor and Co-Director, Program on Aging, Disability, and Long-Term Care, Cecil G. Sheps Center for Health Services Research, University of North Carolina at Chapel Hill, 725 Martin Luther King Jr. Boulevard, Box 7590, Chapel Hill, NC 27599-7590; e-mail: Sheryl_Zimmerman@unc.edu.

Received: October 17, 2015; Accepted:December 2, 2015

doi:10.3928/19404921-20151218-01 
Infections are a common problem in long-term care, with an estimated $12 \%$ of nursing home residents in the United States experiencing an infection at any given time (Dwyer et al., 2013). In addition to causing considerable morbidity and mortality, infections in nursing homes are costly, common causes of hospitalization and death, and associated with antibiotic overuse and the rise of resistant organisms (Boockvar et al., 2005; Strausbaugh \& Joseph, 2000). To help reduce the spread of infections, the Centers for Medicare \& Medicaid Services (CMS) requires registered nursing homes to implement infection prevention and control programs (U.S. Department of Health and Human Services \& CMS, 2014). These programs must include outcome surveillance systems using data on individual cases that can be aggregated to discern rates and patterns of infection within and across nursing homes and to identify priorities for quality improvement. However, the quality of the related data and analyses is often compromised by inconsistencies in definitional criteria and data collection methods (McKibben et al., 2005).

Pneumonia exemplifies the challenges associated with quality improvement efforts related to infection prevention and control. Approximately 2 million episodes of pneumonia are experienced by nursing home residents in the United States every year, resulting in more deaths than from any other infection (Muder, 2000; Quagliarello et al., 2005). Compared to older adults living in the community, the incidence of pneumonia among nursing home residents is approximately 10-fold greater (Quagliarello et al., 2005). Pneumonia is also the leading cause of acute hospitalization for nursing home residents, as approximately one third of cases require hospital admission (Loeb, McGeer, McArthur, Walter, \& Simor, 1999; Muder, 1998). Relatedly, the excess Medicare costs for acute care of pneumonia exceed $\$ 7$ billion annually, with nursing home residents accounting for an estimated $28 \%$ of that expenditure (Naughton \& Mylotte, 2000; Thomas et al., 2012). Despite the importance of this condition, there is no single, definitive strategy that quality improvement personnel or clinical investigators can use to ascertain pneumonia rates in nursing homes. Instead, a number of different approaches have been used.

One approach is to identify all cases that have been clinically diagnosed as pneumonia. However, differentiating pneumonia from other conditions in nursing home populations can be difficult. Nursing home residents often lack the classical clinical findings of pneumonia, such as fever, shortness of breath, cough, or rales, and instead may present with nonspecific or atypical signs and symptoms, such as delirium, loss of appetite, falling, or general weakness (High et al., 2009; Mehr et al., 2001). Further, conditions such as chronic obstructive pulmonary disease or congestive heart failure can at times obscure the diagnostic picture (Dosa, 2006). In addition, many nursing home residents are unable to provide a meaningful history due to cognitive impairment (El Solh, 2009). Consequently, there is no consistent "gold standard" definition of pneumonia using clinical criteria.

Another approach is to document cases that meet surveillance definitions. One such approach is to use the modified McGeer criteria, which comprise (a) chest radiography results showing the presence of a new infiltrate; (b) at least one respiratory criterion (e.g., new or increased cough or sputum production); and (c) at least one constitutional criterion (e.g., fever, acute mental status change) (Stone et al., 2012). However, radiographic evidence is not always available, nor are both respiratory and constitutional criteria recorded consistently in medical records.

A third approach is to document all episodes of antimicrobial therapy for presumed lower respiratory infections that demonstrate clinical findings strongly suggestive of pneumonia. Federal guidelines require nursing home staff to monitor infections, which is typically done by identifying new antibiotic prescribing and having a nurse review the medical record and assign a diagnosis. However, the method of assigning a diagnosis often is idiosyncratic and not guideline-based, and in some instances, a diagnosis of pneumonia is assigned only if confirmed by chest radiography, which may introduce additional inconsistency in that considerable variation has been noted in the use and accuracy of diagnostic radiography across nursing homes (Loeb et al., 2006; Mubareka et al., 2007). Further, the approach of case identification based on a prescription assumes that all pneumonia cases have been adequately identified and treated, but advance directives or clinical decisions at the time of diagnosis may in some cases preclude the use of antibiotic agents (Khandelwal, Lathren, \& Sloane, 2012).

These varying approaches may generate different estimates of pneumonia incidence. For example, a Canadian study found statistically different mortality rates among residents with lower respiratory tract infection in 21 nursing homes using different methods of identification: the mortality rate was $8 \%$ when monitored according to antibiotic treatment; $14 \%$ according to a recorded medical diagnosis; and 9\% using chest $\mathrm{x}$-ray results (Nicolle et al., 2008). Thus, a clear need exists to identify a practical and consistent method of identifying nursing home cases 
of pneumonia for use in surveillance, quality improvement, and research.

In response, the current study compared incidence rates generated by three different approaches to identifying pneumonia: (a) clinical diagnosis of pneumonia as recorded in a physician, nurse, or hospital discharge note; (b) modified McGeer criteria (excluding constitutional symptoms); and (c) prescription of an antibiotic agent for presumed pneumonia plus one or more clinical signs that are highly suggestive of pneumonia. All three approaches rely on information recorded in nursing home charts. Results indicate a robust and pragmatic method for ascertaining pneumonia incidence using nursing home records.

\section{METHOD}

Administrators from 21 nursing homes that evidenced a history of high rates of hospitalization for pneumonia (CMS, 2015; The Dartmouth Atlas of Health Care, 2015; LTCFocus.org, 2015) were identified from six counties in North Carolina and invited to participate in the project. Sixteen $(76 \%)$ administrators agreed to participate. The size of their nursing homes ranged from 62 to 150 beds (mean $=106.6$ beds, $S D=25.1$ beds).

Two highly trained and experienced members of the research team visited each home and retrospectively reviewed 6 months of medical record data (for the period July 1, 2013 through December 31, 2013) for up to 80 randomly selected residents living in the home as of January 1, 2014 (excluding those receiving short-term rehabilitation). To assure coding reliability, a detailed abstracting protocol was developed, and at the beginning of data collection, the two researchers reviewed the same five charts, compared codes, and resolved differences. To assure ongoing agreement throughout the chart review process, they brought questionable cases to the full research team for review and input. In total, 1,119 records were reviewed, ranging from 55 to 81 records per home (mean $=74$ records, $S D=8$ records). Information abstracted from records included resident demographic characteristics, clinical indicators related to a potential case of pneumonia, and hospitalization. Data were obtained from review of all physician notes, nurses' notes, and hospital discharge summaries.

An incident case of pneumonia was defined as one separated from a prior pneumonia case by at least 21 days. In addition, it had to meet one of the three following criteria: (a) clinical diagnosis: written documentation of a diagnosis of pneumonia recorded in a physician, nurse, or hospital discharge note (van der Steen, Ooms, Mehr, van der Wal, \& Ribbe, 2002); (b) modified McGeer criteria (the current authors excluded the "constitutional criteria" portion of the modified McGeer criteria because nursing home documentation does not allow accurate assessment as to whether these criteria have been met): chest $\mathrm{x}$-ray with infiltrate plus either new or increased cough, new or increased sputum production, oxygen saturation $<94 \%$ on room air or reduction in oxygen saturation $\geq 4 \%$ from baseline, new or changed lung examination abnormalities (e.g., rales, crackles, increased fremitus), pleuritic chest pain, and/or respiratory rate $\geq 25$ breaths per minute (Stone et al., 2012); or (c) prescription of an antibiotic drug that is indicated for pneumonia plus one or more of the following signs/ symptoms that are relatively specific for pneumonia: oxygen saturation $<94 \%$ on room air or reduced by $\geq 4 \%$ from baseline, and/or respiratory rate $\geq 25$ breaths per minute (Mubareka et al., 2007). When any one of the criteria for pneumonia were met, all characteristics of that event (as listed above) were abstracted.

Data were double entered into Microsoft Access databases and compared for errors of logic and entry using SAS 9.2 software. Analyses were completed using SPSS 18 for Windows. Descriptive analyses determined the number and percent of events that met criteria for pneumonia based on any of the three definitions and were used to characterize incident cases; in addition, pneumonia rates per 1,000 resident days were calculated. Characteristics and rates were described for all events and sets of events defined by different combinations of criteria (i.e., events defined both by diagnosis and infiltrate plus at least one sign or symptom). All materials and procedures were reviewed and approved by the Institutional Review Board of the University of North Carolina at Chapel Hill.

\section{RESULTS}

Of 1,089 records that included complete data (30 records were excluded due to absent data on key variables), 108 (9.9\%) contained a diagnosis of pneumonia as determined by one or more of the current authors' identification methods. The rate of pneumonia per 1,000 resident days ranged from 0.71 (based on clinical diagnosis) to 0.56 (based on modified McGeer criteria) to 0.32 (based on antibiotic prescription and one or more relatively specific clinical sign). When antibiotic agents were prescribed, the most common drugs chosen were levofloxacin (55\%), azithromycin (17\%), and ciprofloxacin (7\%).

Comparison of the three pneumonia detection strategies is displayed graphically in the Figure. The criterion of clinical diagnosis captured 107 (99.1\%) of 108 pneumonia cases determined by any of the three criteria; the one ex- 


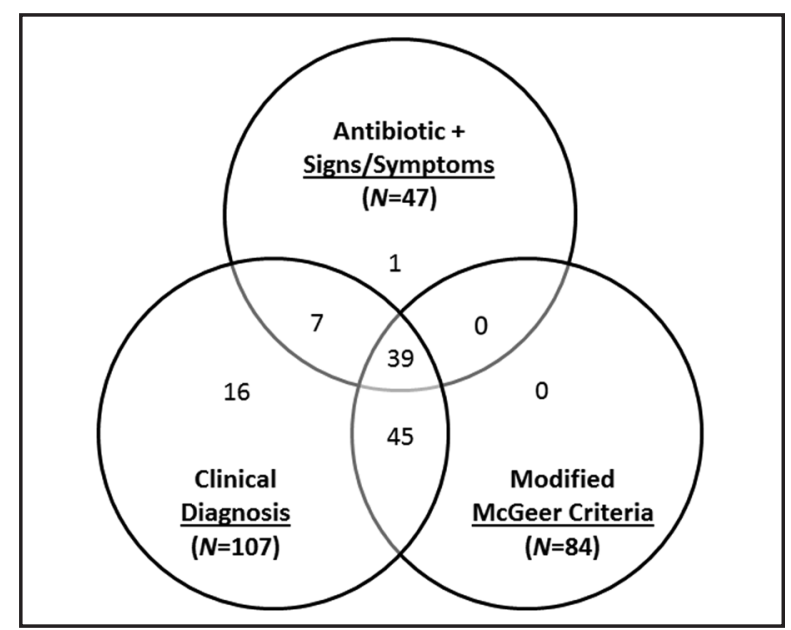

Figure. Venn diagram indicating diagnosis of pneumonia by three criteria.

ception was prescribed an antibiotic agent and met related criteria but did not also have a diagnosis of pneumonia. All cases that met the infiltrate criteria were also recognized based on diagnosis.

The Table provides the demographic characteristics, clinical indicators, and hospitalization data related to cases with any of the three criteria, and all combinations of the criteria. Fever was often absent. Specifically, 31\% of incident cases (by any of the three criteria) had a recorded temperature $\geq 99^{\circ} \mathrm{F}$, and $11 \%$ had a temperature $\geq 100.4^{\circ} \mathrm{F}$.

\section{DISCUSSION}

Scientific evidence and practice guidelines suggest that pneumonia should be considered both a potentially preventable condition and a cause of potentially preventable hospitalization. Consequently, it is not surprising that pneumonia has been identified as a target condition for quality monitoring and improvement efforts in longterm care settings, making accurate and consistent case identification critical. The current study compared three methods for identifying cases of pneumonia from nursing home records and found that one simple methodlooking for a pneumonia diagnosis in the medical or nursing notes-captured $99 \%$ of cases identified by any of the three methods. Consequently, nursing home staff may feel confident to use diagnosis as a sensitive indicator to guide their quality improvement efforts.

It could be argued that a documented clinical diagnosis might undercount some cases, for example, when pneumonia is suspected and antibiotic agents are prescribed over the telephone without a diagnosis being recorded. However, as demonstrated in the Figure, the antibiotic plus clinical signs method added only one case (1\%) over and above the diagnosis method, and the modified McGeer method added no cases. Although this is a reassuring finding, some cases may be missed because incomplete documentation in nursing home records could limit detection via these other methods as well. For example, the absence of documented symptoms (e.g., cough in 33\% of incident cases, new or increased sputum in $57 \%$ of cases, and a new or changed lung abnormality in $61 \%$ of cases) raises the possibility that inadequate documentation may limit the applicability of the McGeer criteria. However, data from a previous study that used research nurses to evaluate 2,334 cases of suspected respiratory infection found cough in $84 \%$, sputum production in $30 \%$, and crackles on lung examination in $66 \%$ (Mehr et al., 2001)-figures that are of a comparable order of magnitude to those the current authors' observed, suggesting that under-documentation may be less common than feared.

Over-documentation based on diagnosis alone is another possibility, especially because the modified McGeer criteria, which were designed for disease surveillance purposes, such as quality monitoring, identified only 84 (79\%) of 107 clinically diagnosed pneumonia cases. On the other hand, it could be argued that the modified McGeer criteria are too strict because they require a chest $\mathrm{x}$-ray (Stone et al., 2012), a contention that is supported by the fact that eight additional cases were identified who received an antibiotic agent and had either a drop in oxygen saturation or a respiratory rate $>25$ breaths per minute, both of which are fairly specific signs of pneumonia (Mubareka et al., 2007). However, nine of 16 cases identified on the basis of clinical diagnosis alone had a chest x-ray, somewhat attenuating this concern. Another issue that could lead to over-diagnosis of pneumonia is the known imprecision of a finding of infiltrate on chest $\mathrm{x}$-ray, which in this population may represent atelectasis, scarring from previous illness, or poor technique (as most nursing home chest $\mathrm{x}$-rays are portable films) (Loeb et al., 2006). Finally, an additional cause for concern regarding over-diagnosis is the fact that only $31 \%$ of cases diagnosed as pneumonia met the low, but nursing home-appropriate, fever threshold of $99^{\circ} \mathrm{F}$ (Sloane et al., 2014).

The 16 cases identified by clinical diagnosis alone (15\% of all cases) represent an interesting group. All were treated with antibiotic agents, $56 \%$ had a chest $\mathrm{x}$-ray, and they evidenced lower rates of hospitalization for pneumonia and days hospitalized for pneumonia than cases identified through any other method. Although one might question whether the diagnosis was justified, it may also be hypothesized that these cases were identified early in the disease course and treated before they became more severe. 


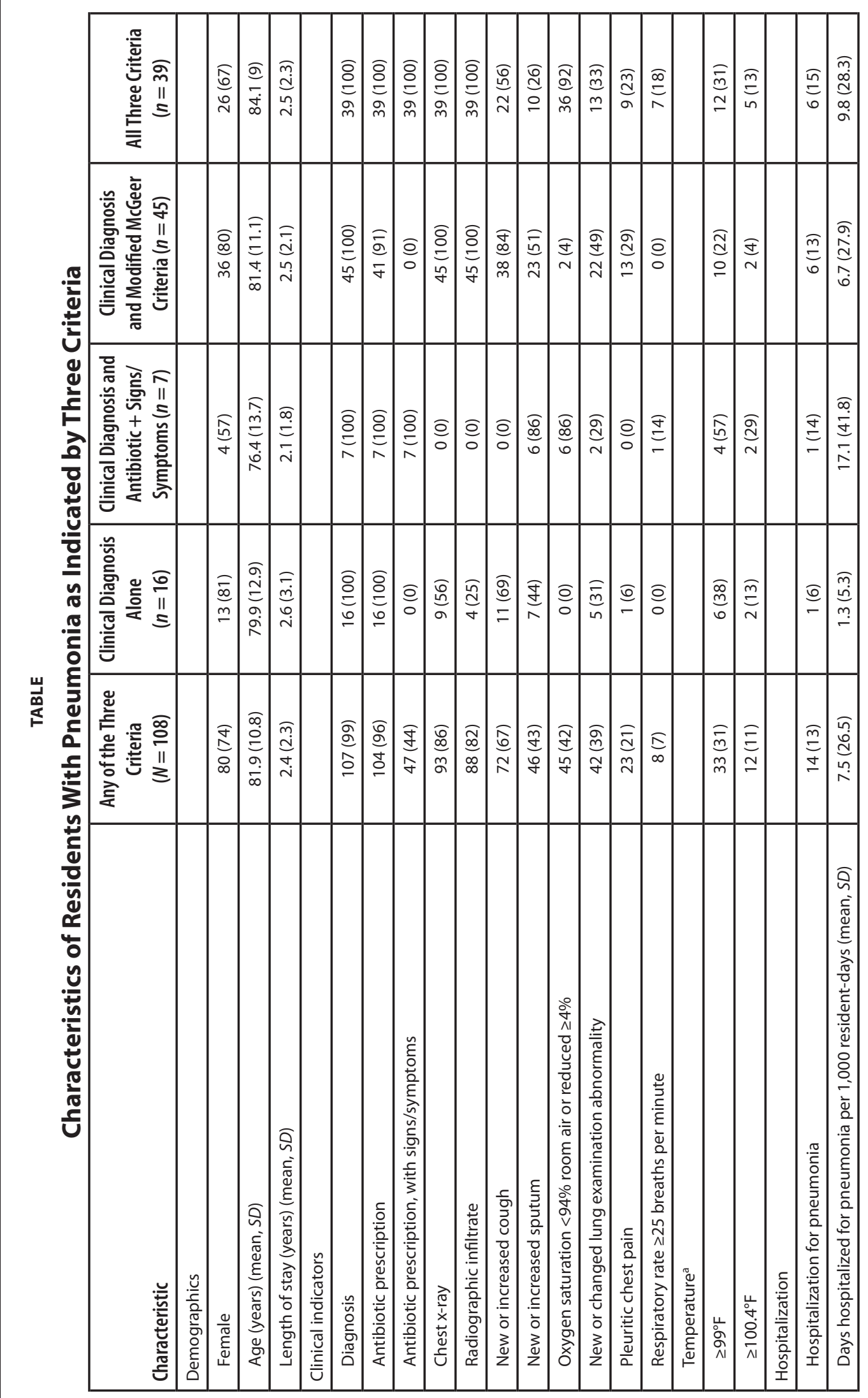

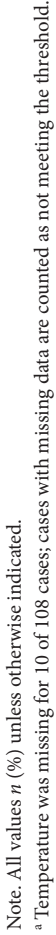




\section{CONCLUSION}

Although the extent of under-identification, and more so over-identification, cannot be ascertained, data obtained for 1,089 residents in 16 nursing homes in North Carolina found that a diagnosis of pneumonia as written in a physician's note, nurse's note, or on a hospital discharge summary is a highly reliable and inclusive method to ascertain pneumonia. It is possible that the quality of documentation in these nursing homes may differ from that in other nursing homes, but if so, it might be assumed that documentation practices (whether more or less complete) will be consistent across the three criteria, thereby generating similar results. Consequently, a diagnosis of pneumonia as recorded on a nursing home chart is useful to guide surveillance and quality improvement and inform research.

\section{REFERENCES}

Boockvar, K.S., Gruber-Baldini, A.L., Burton, L., Zimmerman, S., May, C., \& Magaziner, J. (2005). Outcomes of infection in nursing home residents with and without early hospital transfer. Journal of the American Geriatrics Society, 53, 590-596.

Centers for Medicare \& Medicaid Services. (2015). Community-based care transitions program. Retrieved from https://innovation.cms. gov/initiatives/CCTP/?itemID=CMS1239313

The Dartmouth Atlas of Health Care. (2015). Data by region. Retrieved from http://www.dartmouthatlas.org/data/region

Dosa, D. (2006). Should I hospitalize my resident with nursing homeacquired pneumonia? Journal of the American Medical Directors Association, 7(Suppl. 3), S74-S80.

Dwyer, L.L., Harris-Kojetin, L.D., Valverde, R.H., Frazier, J.M., Simon, A.E., Stone, N.D., \& Thompson, N.D. (2013). Infections in longterm care populations in the United States. Journal of the American Geriatrics Society, 61, 342-349.

El Solh, A.A. (2009). Nursing home-acquired pneumonia. Seminars in Respiratory and Critical Care Medicine, 30, 16-25. doi:10.1055/s-0028-1119805

High, K.P., Bradley, S.F., Gravenstein, S., Mehr, D.R., Quagliarello, V.J., Richards, C., \& Yoshikawa, T.T. (2009). Clinical practice guideline for the evaluation of fever and infection in older adult residents of long-term care facilities: 2008 update by the Infectious Diseases Society of America. Clinical Infectious Diseases, 48, 149171. doi:10.1086/595683

Khandelwal, C., Lathren, C., \& Sloane, P.D. (2012). Ten clinical situations in long-term care for which antibiotics are often prescribed but are rarely necessary. Annals of Long-Term Care, 20, 23-29.

Loeb, M., McGeer, A., McArthur, M., Walter, S., \& Simor, A.E. (1999). Risk factors for pneumonia and other lower respiratory tract infections in elderly residents of long-term care facilities. Archives of Internal Medicine, 159, 2058-2064.

Loeb, M.B., Carusone, S.B.C., Marrie, T.J., Brazil, K., Krueger, P., Lohfeld, L.,...Walter, S.D. (2006). Interobserver reliability of radiologists' interpretations of mobile chest radiographs for nursing home-acquired pneumonia. Journal of the American Medical Directors Association, 7, 416-419.
LTCFocus.org. (2015). Long-term care: Facts on care in the US. Retrieved from http://www.ltcfocus.org

McKibben, L., Horan, T.C., Tokars, J.I., Fowler, G., Cardo, D.M., Pearson, M.L., \& Brennan, P.J. (2005). Guidance on public reporting of healthcare-associated infections: Recommendations of the Healthcare Infection Control Practices Advisory Committee. American Journal of Infection Control, 33, 217-226.

Mehr, D.R., Binder, E.F., Kruse, R.L., Zweig, S.C., Madsen, R.W., \& D'Agostino, R.B. (2001). Clinical findings associated with radiographic pneumonia in nursing home residents. Journal of Family Practice, 50, 931-937.

Mubareka, S., Duckworth, H., Cheang, M., Loeb, M., Simor, A., Liu, B.,... Nicolle, L.E. (2007). Use of diagnostic tests for lower respiratory infection in long-term care facilities. Journal of the American Geriatrics Society, 55, 1365-1370.

Muder, R.R. (1998). Pneumonia in residents of long-term care facilities: Epidemiology, etiology, management, and prevention. American Journal of Medicine, 105, 319-330.

Muder, R.R. (2000). Management of nursing home-acquired pneumonia: Unresolved issues and priorities for future investigation. Journal of the American Geriatrics Society, 48, 95-96.

Naughton, B.J., \& Mylotte, J. (2000). Treatment guideline for nursing home-acquired pneumonia based on community practice. Journal of the American Geriatrics Society, 48, 82-88.

Nicolle, L.E., Mubareka, S., Simor, A., Liu, B., McNeil, S., Lewis, D.,... Loeb, M. (2008). Variation in mortality rates among long-term care facilities for residents with lower respiratory tract infection. Infection Control and Hospital Epidemiology, 29, 754-759. doi: $10.1086 / 590123$

Quagliarello, V., Ginter, S., Han, L., Van Ness, P., Allore, H., \& Tinetti, M. (2005). Modifiable risk factors for nursing home-acquired pneumonia. Clinical Infectious Diseases, 40, 1-6.

Sloane, P.D., Kistler, C., Mitchell, C.M., Beeber, A.S., Bertrand, R.M., Edwards, A.S.,...Zimmerman, S. (2014). Role of body temperature in diagnosing bacterial infection in nursing home residents. Journal of the American Geriatrics Society, 62, 135-140.

Stone, N.D., Ashraf, M.S., Calder, J., Crnich, C.J., Crossley, K., Drinka, P.J.,...Bradley, S.F. (2012). Surveillance definitions of infections in long-term care facilities: Revisiting the McGeer Criteria. Infection Control and Hospital Epidemiology, 33, 965-977. doi: $10.1086 / 667743$

Strausbaugh, L.J., \& Joseph, C.L. (2000). The burden of infection in long-term care. Infection Control and Hospital Epidemiology, 21, 674-679.

Thomas, C.P., Ryan, M., Chapman, J.D., Stason, W.B., Tompkins, C.P., Suaya, J.A.,...Shephard, D.S. (2012). Incidence and cost of pneumonia in Medicare beneficiaries. Chest, 142, 973-981.

U.S. Department of Health and Human Services, \& Centers for Medicare \& Medicaid Services. (2014, April 11). CMS manual system (Pub. 100-07 State Operations Provider Certification). Retrieved from https://www1b.cms.gov/Regulations-and-Guidance/ Guidance/Transmittals/Downloads/R108SOMA.pdf

van der Steen, J.T., Ooms, M.E., Mehr, D.R., van der Wal, G., \& Ribbe, M.W. (2002). Severe dementia and adverse outcomes of nursing home-acquired pneumonia: Evidence for mediation by functional and pathophysiological decline. Journal of the American Geriatrics Society, 50, 439-448. 